\title{
PERCEPTION ET ENJEUX DU DECOUPAGE TERRITORIAL EN REPUBLIQUE DEMOCRATIQUE DU CONGO.
}

\author{
Cas de la Province du Haut-Lomami
}

Par NKULU KILUMBA BOSHWAA, assistant à l'Université de Kamina

\section{RESUME}

L'occasion faisant le larron, celle qu'offre la décentralisation territoriale par le biais du découpage du territoire congolais est perceptible de deux façons, parce que l'enjeu est aléatoire.

C'est cet aléa qui crée dans le chef de certains ressortissants du Haut-Lomami un sentiment conservatiste d'un régionalisme traditionnel (provincialisme unitaire), se basant ainsi sur les ressources financières modiques comme raison d'être attaché au sud qui est utile (financièrement viable), car le Haut Lomami est un Katanga inutile (Financièrement moins viable) et au critère subjectif sur lequel l'on s'est fondé pour opérer le découpage favorisant la discrimination et le tribalisme, la deuxième façon de voir les choses est positive, revigorée non seulement par le rapprochement des dirigeants des dirigés, en vue d'assurer une meilleur prise en compte des problèmes de ces derniers et y apporter des solutions rapides et adaptées à leurs besoins... mais aussi et surtout viabiliser ce Katanga inutile (Haut-Lomami) par le solutionnement adapté aux problèmes majeurs comme la pauvreté, le chômage...

Comme dans une démarche démocratique l'abstention ne manque jamais, une catégorie truande ronge aussi le peuple mitan du Haut-Lomami, la future province, même si la majeure partie de la population est prête pour accueillir la province et est convaincu de tous les atouts préalables à la réussite de son installation.

Le Haut Lomami est une province pentagone du Congo, composée de 5 territoires d'une même tribu, force qu'on ne trouvera pas facilement ailleurs, symbole d'unité pour réussir. Province aux ressources naturelles encore vierges, à vocation maritime, agropastorale et minérale.

\section{INTRODUCTION}

La population du Haut Lomami est, quel que soit la volonté du constituant ou mieux, la mise en place d'une constitution adoptée par voie référendaire, partagée sur le découpage du Haut Lomami comme province. Des différences résultent de la modicité des ressources financières et du manque d'infrastructures de base (bâtiments devant abriter l'administration, les routes,...) 
Ainsi, advient la nécessité de faire un contour explicatif du découpage territorial et la décentralisation avant d'aborder l'étude sur le plan historique et la perception négative et ses enjeux ainsi que la perception positive et ses enjeux, description pragmatique des données collectées par enquête directe.

Le découpage est la subdivision du territoire d'un Etat en des entités décentralisées en vue d'une politique de proximité.

La décentralisation, quant à elle, est un transfert de pouvoir de l'Etat vers des personnes morales de droit public distinct. C'est un processus d'aménagement de l'Etat unitaire qui consiste à transférer les compétences administratives de l'Etat vers des entités ou collectivités locales distinctes de ce dernier

Le découpage territorial est alors une disposition constitutionnelle qui vient renforcer la décentralisation comme une TECHNIQUE DE DEVELOPPEMENT pour rendre efficace la gestion décentralisée.

\section{HISTORIQUE DE DECOUPAGES TERRITORIAUX EN RDC}

\section{I.1. La période de l'Etat indépendant du Congo (1885-1908)}

L'Etat indépendant du Congo (EIC) n'a d'abord eu aucune organisation territoriale systématique, le roi Souverain LEOPOLD II, avant même la reconnaissance de l'Etat, avait mis sur s pied à Bruxelles une administration organisée en trois départements qui, jusqu'en 1894 n'était même pas subordonnée à une autre autorité hiérarchique et celle du Roi.

Dès 1884 il avait nommé un Anglais FRANCIS DE WINTON, administrateur de l'association internationale Africaine à VIVI. Mais l'EIC n'a jamais eu de constitution et le gouverneur général qui reçut ce titre par décret du 30 juillet 1886, n'avait d'autre pouvoir que celui que le Roi lui déléguait.

Un premier découpage général en onze districts, dont cinq se trouvent entre l'océan atlantique et le Kwango, fut décrété le 1 Août 1888, mais les commissaires de districts n'avaient pas tous le même pouvoir.

Des zones furent ensuite créées dans certains districts, mais elles n'en étaient pas une subdivision systématique et certains chefs de zones, dans les régions troublées ou menacées, reçurent de pouvoirs égaux ou supérieurs à ceux du commissaire de district dans la juridiction où ils se trouvaient.

Le régime de l'EIC était donc extrêmement centralisé à Bruxelles et les agents localement en place n'avaient de compte à rendre qu'à Bruxelles.

\section{I.2. La période coloniale}

A partir de 1908 à 1933, un des soucis de rédacteurs de la charte coloniale qui régit le Congo Belge du 18 Octobre 1908 au 30 Juin 1960 fut d'alléger l'administration métropolitaine de la colonie. Une mesure prise dans ce but fut de réserver au seul gouverneur général le droit de correspondre avec Bruxelles. 
Un autre souci fut d'organiser le contrôle de l'administration non seulement par le parlement Belge auquel un rapport annuel devait être fourni par le ministre de colonie, mais par de contrôle hiérarchique des agents au Congo.

Le nombre de niveau hiérarchique fut étendu en subdivisant d'abord le district en territoires, à partir de 1912 et en les regroupant en suite en quatre provinces à partir de 1914. A partir des années 1920, des chefferies, et plus tard des secteurs, deviennent en outre des subdivisions systématiques de territoires.

La subdivision des districts en territoires initiée en 1912-1914 avait aussi pour objectif la prise de contrôle plus étroite de l'espace et de populations.

Il y avait 184 territoires au terme de cette première organisation et leur nombre resta de même ordre jusqu'en 1931 lorsque la crise économique de 1929 en imposant une réduction en fonction de contrainte budgétaire.

En 1922, le gouverneur général reçut le pouvoir de fixer le nombre et les limites des districts que le roi s'était au paravent réservé. Les gouverneurs de la province orientale et du Katanga réussirent à donner une telle impulsion aux autorités de leurs circonscriptions qu'ils firent redouter.

Hormis les réformes au cours desquelles les districts vont varier, leur nombre est passé de manière suivante :

- 12 en 1910

- 22 en 1912

- 21 en 1922

Le Congo va connaitre trois subdivisions territoriales majeures, celles de 1914,1924 et 1933.

La réforme de 1914 fut initiée et opérée par l'arrêté royal du 28 juillet 1914 qui divisa le pays en 22 districts dont le gouvernement siégeait à BOMA et deux vice-gouverneurs siégeant l'un à Elisabethville et l'autre à Stanley ville.

Celle de 1924 regroupa les districts en quatre provinces : le Katanga, le Congo -Kasaï, l'équateur et la province orientale. Les provinces seront subdivisées en districts et en territoires, en zones, en secteurs et en postes.

Enfin, celle de 1933, c'est la plus grande réforme sous le Congo-belge qui s'opéra en deux temps et à deux niveaux :

Dans un premier temps, au niveau de l'organisation territoriale et administrative de type européen, la réforme a été opérée par l'arrêté royal du 29 juin 1933. Dans un second temps, au niveau des entités de base, la réforme sera réalisée par le décret du 05 décembre 1933 sur les circonscriptions indigènes et celles-ci ne comprenaient que les chefferies et les secteurs à l'exception de chefs coutumiers, régis à cette époque par le décret du 23 novembre 1931.

En effet, au terme des dispositions de l'article premier de l'arrêté royal du 29 juin 1933 relatif à la constitution de chefs-lieux et limites de provinces, le nombre de provinces passa de 4 à 6 , à savoirs : 
- Coquilhatville, équateur après 1947 (districts : Haut-Congo, équateur et Tshikapa);

- Elisabethville, Katanga après 1947 avec 4 districts : Haut-Katanga, haut Lomami, Lualaba et Tanganyika.

- Costermansville, Kivu après 1947 avec 3 districts : Nord-Kivu, Sud-Kivu et Maniema;

- Léopoldville avec 3 districts : le moyen-Congo, le Bas-Congo, lac Léopold II;

- Lusambo, Kasaï après 1947, trois districts : le Kasaï, le Sankuru, le Kabinda;

- Stanley ville, province orientale après 1947 avec 4 districts : Stanley ville, Bas-Uélé, Haut-Uélé et Ituri.

\section{I.3. La période postcoloniale}

Le territoire congolais demeurait diviser en six provinces hérités du Congo-belge, jouissant cependant d'une très large autonomie car cette forme dotait l'Etat congolais d'une forme fédérale.

Au cours de l'année 1962, deux lois vont intervenir, celle du 09 mars et celle du 27 avril, modifiant l'article 7 de la loi fondamentale concernant les provinces et fixait les critères de leur création pour passer ainsi le nombre de provinces de 6 à 21. Ce qui engendre le terme populaire et ironique de « provincettes »

Une autre réforme fut apportée par l'ordonnance -loi n ${ }^{\circ} 031$ du 20 juillet 1988, c'est fut le découpage territorial mais dont l'œuvre était inachevée, qui érigea les anciennes sous régions de KIVU en trois nouvelles provinces.

Le nombre de province passa jusqu'à dix plus la ville de Kinshasa il s'agissait de :

- Nord Kivu;

- Sud Kivu;

- Maniema;

- Bandundu

- Equateur;

- Bas-Congo;

- Kasaï-Oriental;

- Kasaï-Occidental;

- Katanga;

- La province Oriental

- Plus la ville de Kinshasa, qui a le statut de la province

Sous la troisième république, la constitution énumère les futures provinces dont celle du Haut-Lomami.

La RDC est composée de la ville de Kinshasa et 25 provinces dotées des personnalités juridiques. Ces provinces sont :

- Bas-Uélé,

- Equateur,

- Haut-Lomami, 
- Haut-Katanga,

- Haut Uélé,

- Ituri,

- Kasaï,

- Kasaï oriental,

- Congo central,

- Kwango,

- Kwilu,

- Lomami,

- Lualaba,

- Kasaï central,

- Maï-Ndombe,

- Maniema,

- Mongola,

- Nord Kivu,

- Nord Ubangi,

- Sankuru,

- Sud-Kivu,

- Sud-Ubangi,

- Tanganyika,

- Tshopo,

- Tshuapa.

Ainsi, le découpage n'est ni une innovation de l'actuelle constitution ni de l'actuelle république (la troisième), l'Etat indépendante du Congo l'avait prévu, découpage général en onze districts par décret du 1 août 1888, sous la colonisation plusieurs réformes ont été enregistrées, en 1910, 1912 et 1922, mais les plus grandes étaient celles de 1914, 1924 et surtout celle de 1933 qui ramena les provinces de 4 à 6 . Sous l'empire de la deuxième république, deux lois intervenant en 1962 pour modifier l'article 7 de la loi fondamentale et qui créa 21 provincettes.

Le dernier découpage en date est celui intervenu avec la réforme de 1988. Etant inachevée et souvent l'idée de non concrétisée, voilà l'occasion sous l'actuelle constitution de parachever ce qui était, qui a été et qui est.

\section{PERCEPTION NEGATIVE ET SES ENJEUX}

Les ressortissants du district du Haut Lomami, future province, ont la liberté d'expression à l'instar des autres ressortissants d'autres districts en dépit de cette contextualisation (du découpage).

Vivant sur place et connaissant toutes les réalités, certaines personnes se rendent hostiles au découpage. Elles avancent des raisons aussi nombreuses que fondées, ces raisons sont soit liées au critère fondamental sur lequel le constituant s'est basé pour y arriver, soit 
basées au manque d'infrastructures de base, soit favorisant le tribalisme, soit à l'impréparation et absence du recensement de la population,...

Certes, le découpage est une opportunité et offre des sérieux avantages, la percevoir négativement ne signifie pas la méconnaitre, refuser des avantages qu'il offre, être inconstitutionnel dans le mesure ou la constitution le prévoit mais parce que ce district, le Haut Lomami, n'est pas déjà préparé, au moins pour ce moment.

Et la faiblesse majeure que présente l'actuel découpage est, qu'il a pour socle, la transformation des anciens districts en provinces, excepté le Bas-Congo; c'est ce que on appelle découpage sur des bases ou critères subjectifs ou encore ethniques.

L'impression est que l'on n'a pas tenu compte des critères objectifs de viabilité, critères qui encourageraient la lutte contre le tribalisme, or le District du Haut-Lomami, future province ne va aligner que les luba de :

- Malemba Nkulu,

- Bukama,

- Kabongo,

- Kaniama

- Kamina, critère subjectif favorisant le tribalisme.

Le Haut-Lomami sera une province essentiellement Luba où l'intégration d'autres langues conduira soit à la discrimination, soit au tribalisme, alors pourquoi faire pour un pays qui se dit uni et dont les autres langues font partie du patrimoine culturel congolais?

Il n'y a eu ni recensement des populations, ni consultations de sentiments de vouloir vivre collectif, ni formation des experts et animateurs de la territoriale.

Bien plus, on n'a pas non plus tenu compte des infrastructures administratives, des redéploiements des fonctionnaires, des moyens financiers à mobiliser pour appliquer la décentralisation, du renforcement des capacités de gestion, cela a pour conséquences certaines que la province (Haut Lomami) manque presque tout.

L'on se demande alors si le seul critère qui consiste à faire des anciens districts des provinces suffit pour asseoir le découpage territorial, car la province orientale, la plus vaste de 11 provinces du pays ne sera découpée qu'en quatre provinces;

- Le Katanga avec une superficie de $496.877 \mathrm{Km}^{2}$ et plus au moins 8.949 .000 habitants n'alignera que quatre provinces alors que

- celle de l'Equateur avec $403.282 \mathrm{Km}^{2}$ et 6.414 .000 habitants en disposera cinq.

- L'actuelle province du Bandundu avec $295.580 \mathrm{Km}^{2}$ et 7.018 .000 habitants ne sera subdivisée qu'en trois provinces au même titre que le Kasaï Oriental deux fois moins étendu (169.886 $\mathrm{Km}^{2}$ et 5.421 .000 habitants)

La configuration du Haut-Lomami comme d'ailleurs celle des autres provinces repose sur des critères purement ethniques ou subjectifs qui amènent le tribalisme et la discrimination. En plus ce critère est injuste par application des données statistiques susmentionnées. 


\section{PERCEPTION POSITIVE ET SES ENJEUX}

De prime abord, le découpage territorial de la République démocratique du Congo est une voie constitutionnelle, entant que telle, elle doit être suivie et menée à bout et une opportunité qui offre des avantages si l'on prend en compte les dimensions continentales de notre pays qui se situent à $2.345 .420 \mathrm{Km}^{2}$. Plusieurs pays moins vastes que le nôtre ont recouru à cette technique pour une meilleure gestion de leur espace national, à titre d'exemple, nous citons :

- la France qui, avec $550.000 \mathrm{Km}^{2}$ environs quatre fois moins que la RDC, est découpée en 100 départements ou provinces, en 341 arrondissement ou territoires, en 4.039 cantons ou secteurs/chefferies et en 36.782 communes ou groupements;

- l'Allemagne qui, avec $357.050 \mathrm{Km}^{2}$ environs sept fois moins que la RDC, moins que le Province du Katanga (dont le Haut-Lomami sera détaché), est pourtant découpé en 16 régions ou länder ou provinces, en 26 districts ou territoires et en 16.127 communes (secteurs ou chefferies).

Vue sous cet angle, la décentralisation a l'avantage de rapprocher l'Administration des administrés, d'associer les communautés de base dans la gestion de la cité à travers des organes élus, dotés de pouvoirs spécifiques propres dans des matières telle que définies dans la constitution.

En tout cas ces dimensions méritent d'être découpées pour prétendre à un développement rapide.

Pour rappel, la province du Katanga avec ses $496877 \mathrm{Km}^{2}$ est loin plus étendue que le japon $\left(377801 \mathrm{Km}^{2}\right)$, la Côte-d'Ivoire $\left(322.462 \mathrm{Km}^{2}\right)$, l'Italie $\left(301.278 \mathrm{Km}^{2}\right)$ et même la simple ville de Kinshasa, avec ses $9.965 \mathrm{Km}^{2}$, est trois fois plus étendue que le Luxemburg $\left(2.586 \mathrm{Km}^{2}\right)$. Le découpage pour la RDC tombe à point nommé pour répondre à plusieurs objectifs.

Il facilitera le rapprochement des dirigeants des dirigés, en vue d'assurer une meilleure prise en compte des problèmes de ces derniers et y apporter des solutions rapides et adaptées à leurs besoins.

Il permet la proximité des intérêts des citoyens, l'implication des citoyens dans la gestion de la chose publique, l'application rapide des sanctions, l'alternance au pouvoir par le biais des élections, le renforcement de la démocratie à partir de la base, transparence dans la gestion de la chose publique grâce au contrôle...(7).

Le Haut-Lomami sera une subdivision territoriale dotée de la personnalité juridique qui, en plus de l'autonomie de gestion et de décision, est habilitée à légiférer sur certaines matières conformément à la loi.

Ce qui permettra de sortir le Haut-Lomami du gouffre de la pauvreté, de relever le Haut-Lomami qui, aux yeux des uns c'est le Katanga inutile, voilà l'occasion, aussi par le biais de la caisse de péréquation, de viabiliser cette partie du nord Katanga, c'est maintenant ou jamais pour résoudre plusieurs problèmes, corriger la situation qui fait de la RDC 
un pays aux provinces trop vastes, plus que plusieurs pays surtout ceux de l'Europe, des provinces aux dimensions des pays difficilement gérables (comme province).

Le découpage déclenchera un développement harmonieux du pays dans son ensemble et de ses entités administratives décentralisées, au même moment combattra le chômage et la pauvreté avec la participation de tous à la gestion des affaires publiques dont autre fois l'accès était impossible.

Tous les services auront dans le Haut-Lomami le rang des services aujourd'hui basés dans les actuelles chefs-lieux des provinces (dans l'organisation territoriale de 11 provinces) et tous ces services vont engager des jeunes diplômés, ce qui réfrénera à la fois le chômage et la pauvreté et par ricochet rendra très utile cette partie du pays, alors pourquoi le réfuter, on l'entend et c'est sûr il viendra car c'est constitutionnel, c'est ça le légalisme.

Le découpage sera un sauvetage du Haut-Lomami de la dépendance totale (surtout du nord Katanga). L'heure est arrivée pour que la constitution, au moins pour la décentralisation, soit respectée et appliquée.

\section{CONCLUSION}

Le découpage territorial en RDC n'est ni une innovation de l'actuelle constitution ni du régime en place (la troisième république). Il était, a été et est un processus de toute époque. Sous l'état indépendant du Congo, il était décrété le 01 Août 1888, et organisait onze districts, c'est le tout premier découpage général.

Sous la colonisation il a été consacré par plusieurs réformes, la plus grande a été consacrée par l'arrêté royal du 29 juin 1933, les provinces avaient atteint le nombre de dix. Après la colonisation, l'idée est toujours envie, c'est ainsi qu'il a été créé 21 provincettes au lieu de six, mais le découpage en RDC est une pérégrination et la réussite n'a jamais été de mise. Une autre plus grande réforme fut opérée en 1988 par la loi n031 du 20 Juillet 1988, qui érigea les anciennes sous-régions de Kivu en trois provinces, le nombre passa à dix plus la ville de Kinshasa, onze provinces maintenues jusqu'aujourd'hui, c'est pourquoi l'idée est toujours là, car cette réforme a été inachevée.

Si de toutes les réformes le district du Haut-Lomami n'a jamais eu le statut de province, même celui de provincette, l'occasion est maintenant pour que le Haut-Lomami devienne une province. Cependant la perception de ce découpage territorial en ce qui concerne essentiellement le District du Haut-Lomami est perceptible de deux façons.

Il y en a qui pense que découper le Haut-Lomami aujourd'hui est un découpage à la sauvette, car il n'a ni ressources financières, ni infrastructures de base. Bien plus, ce découpage ne repose que sur le seul critère de transformation des anciens districts en provinces sans tenir compte des critères objectifs comme infrastructures de base, ressources financières... mais l'on constate que le découpage du Haut-Lomami se fonde sur des bases ethniques, facteurs favorisant le tribalisme.

Le Haut-Lomami ne sera composé que des Luba (tous les 5 territoires : Kamina, Bukama, Kabongo, Kaniama et Malemba-Nkulu), parlant la même langue, ce qui conduire à l'in- 
tégration difficile à ceux qui ne savent pas parler Luba et par conséquent, débouchera à la discrimination et au tribalisme.

Alors pourquoi le faire! Cette perceptivité négative du découpage du haut-Lomami repose sur des enjeux négatifs, favorisant l'échec, car le temps n'est pas encore indiqué, au moins présentement. Après la préparation sur multiples plans (infrastructure, critères objectif...) quand le Haut-Lomami sera prêt, le découpage serait salué.

La position dominante est la perception positive, voyant dans le découpage un sauvetage de chômage, de pauvreté et de la dépendance du sud Katanga, ses enjeux sont basés sur l'avantage de rapprocher l'administration des administrés pour trouver rapidement solution aux problèmes, d'associer les communautés de base dans la gestion de la cité à travers les organes élus.

Les dimensions du pays étant continentales, le découpage mérité d'être opéré pour amoindrir l'espace provincial à entretenir, c'est ainsi que le haut-Lomami sera une subdivision dotée de la personnalité juridique, en plus de l'autonomie de gestion et de décision, sera habilitée à légiférer sur certaines matières conformément à la constitution. Il sera question maintenant de viabiliser le Haut-Lomami, de le sortir du gouffre du chômage et de la pauvreté, en vue d'y promouvoir un développement harmonieux.

Le Haut-Lomami est la rare province qui sera composée d'une seule tribu, parlant même langue, symbole d'unité, une arme pour conquérir le combat, car dit-on l'union fait la force. C'est un pentagone congolais riche en ressources naturelles, encore vierges. Bukama et Malemba sont submergés par l'inondation des lacs, ils fournissent beaucoup de poissons; Kabongo, Kaniama et Kamina produisent des maïs, manioc... Kabongo et Malemba donnent de l'huile de palme, Kabongo donne aussi bombwe (le sel), Kamina fournit de la viande; dans tous ces cinq territoires sont disséminés des minerais encore non exploités.

Donc le pentagone congolais est à vocation maritime, agropastorale et minérale. Il y a tous, la réussite est centaine, le découpage est un sauvetage, mais il ne faut pas y aller à la sauvette, il faut commencer par valoriser toutes ces richesses non encore exploitées.

\section{REFERENCES BIBLIOGRAPHIQUES}

0. http://fr.m.wikipédia.org/wiki/décentralisation consulté le 20 Mai 2014

1. LEON DE SAINT MOULIN « l'histoire de l'organisation administrative du Zaïre » in Zaïre-Africa, 1992, n²61, P. 29

2. Arrêté royal du 28 mars 1912 établissant le territoire comme subdivision du District.

3. Ordonnance du 05 Juillet 1912, voir bulletin officiel, P. 1190.

4. KABANGE NTALA, droit administratif,_tome II, Kinshasa, 2001, P. 79

5. Article 2 de la constitution du 18 Février 2006, telle que révisée par la loi n ${ }^{\circ} 011 / 002 \mathrm{du}$ $20 / 01 / 2011$

6. SHAMBUYI TSHIVUNDI MUKWA LUKUSA, la décentralisation en RDC : ce qu'il faut savoir, CDPS, médias, Août 2014, nº50, P. 2 